\title{
Una lectura acerca de la historia del fútbol español: de los comienzos al régimen de Franco
}

\author{
A reading about the history of Spanish football: from the beginning \\ to the Franco regime
}

David Ibarrola david.ibarrola92@gmail.com

http://orcid.org/0000-0001-5154-4938

Universidad de Buenos Aires (Argentina)

\section{Resumen}

El propósito de este trabajo es aproximarse a la comprensión de la historia del fútbol español. Partiendo de la base de que en el fútbol se ponen de manifiesto algunos de los dramas de la sociedad, me propongo ir desenvolviendo esta historia en relación a las características propias de la Nación y su porvenir político, durante el periodo que abarca desde fines del siglo XIX hasta comienzos del franquismo. La profesionalización o la reestructuración de la liga, que también son fenómenos mundiales, marcarán los intentos de una nación "atrasada" de encaminarse hacia una modernidad deportiva, en las que los propios rasgos del "atraso" de pondrán de manifiesto. La idea modernidad y los regionalismos españoles aparecerán en este análisis. Para lograr este objetivo voy a apelar a una serie de trabajos académicos, tanto sobre estos años del deporte español, como de otras naciones, contrastando también brevemente los procesos, con el fin de entender la naturaleza de un fenómeno global en tierras españolas. 
Palabras clave: fútbol; España; profesionalismo; capitalismo.

\section{Abstract}

The purpose of this work is to approach the understanding of the history of Spanish football. Based on the fact that in football some of the dramas of society are revealed, I intend to go developing this history in relation to the characteristics of the nation and its political future during the period from the late nineteenth century until the beginning of Francoism. Thus, the professionalization or restructuring of the league, which are also global phenomena, will mark the attempts of a "backward" nation to move towards a sports modernity, in which the very characteristics of "backwardness" will become clear. The idea of modernity and Spanish regionalisms will appear in this analysis. To achieve this goal I will appeal to a series of academic works, both on these years of Spanish sport, and other nations, also briefly contrasting the processes, in order to understand the nature of a global phenomenon in Spanish lands.

Keywords: football; Spain; professionalism; capitalism.

Altuve señala que "La incorporación directa o indirecta de todo el mundo a una misma lógica de funcionamiento social; la conversión del planeta en un gran mercado mundial es intrínseca a la dinámica de la organización socio-económica" (2002: 21). Esto implica una organización de la vida social, penetrada por la lógica del capital, en íntima relación al mercado. Naturalmente el deporte no escapa a esta realidad. Existen una serie de cuestiones acerca del deporte, específicamente en el fútbol, el deporte que nos convoca, que lo demuestran. Durante las décadas del 20 y del 30 tuvo lugar en el mundo un proceso de profesionalización de la actividad y de reestructuración de la organización de la competencia, del que ni siquiera logró escapar la Unión Soviética, que se encontraba a priori ajena a la lógica del capital (Edelman, 1993).

Es en el desarrollo de este proceso donde estará puesto mi énfasis. La elección del caso, España entre fines del siglo XIX hasta comienzos del franquismo, responde a un interés acerca de las características del mismo, que presenta al igual que en el caso ruso, una revolución durante el período abarcado, solo que en este caso concluye con una derrota. Entender como estos cambios globales en el deporte afectan este lugar del mundo requiere tener presente las 
características de la propia nación europea, no perder de vista los cambios económicos, y también políticos.

Voy a sostener, retomando a Archetti que las transformaciones en el fútbol español, no dejan de reflejar las tensiones políticas experimentadas por aquellos años. Tal como afirma el antropólogo "el fútbol aparece como una 'arena publica' en la que se desarrollan algunos de los dramas de una sociedad y es, por tanto, un vehículo de su cultura" (1985: 5). Pero antes de iniciar cualquier tipo de análisis se hace necesario, especialmente si se afirma que las relaciones entre deporte y capitalismo no pueden ser ignoradas, mencionar ciertas características del país en que nos focalizaremos.

Pierre Broue en su obra La revolución y la guerra en España dice sobre dicha nación: "La España de principios del siglo XX es el arcaísmo de Occidente: en ese mundo que se uniforma, es el islote de las tradiciones y sus amos se vanaglorian de haber sabido mantener su "hispanidad" frente a las corrientes políticas y económicas modernas" (1962: 10). Caracterizada por un pasado imperial "glorioso", hacia el siglo XIX esta nación había perdido todas sus colonias y se encontraba aislada de los grandes movimientos industriales y liberales que conmovían al continente europeo. Nuevamente retomando a Broue:

\begin{abstract}
Las clases del antiguo régimen siguen descomponiéndose, sin que por ello se lleve a cabo la formación de la nueva sociedad burguesa en gestación. El retardo del desarrollo capitalista, el encogimiento de las relaciones económicas frenan la formación de la nación, refuerzan las tendencias centrífugas y el separatismo de las provincias: los empresarios del País Vasco y de Cataluña que, en el siglo XIX, se beneficiaron con un desarrollo industrial limitado, soportan de mal grado, pero sin tener fuerza para sacudírselo, el yugo de la oligarquía castellana (1962: 10).
\end{abstract}

Las masas campesinas, que estaban atravesando un súbito proceso de proletarización, estallaban frecuentemente en importantes rebeliones. Los recientes vínculos con el mundo rural, hacían solidos los lazos que unían a la clase obrera, que era una de las más combativas del momento. Sin lugar a dudas era una sociedad compleja.

De conjunto, estamos ante un desarrollo industrial lento, apenas saliendo del periodo de manufacturas y en lugares geográficos circunscriptos. La concentración de la industria se realizaba con un ritmo muy lento: sólo la metalurgia del País Vasco presenta todos los rasgos de la gran industria capitalista moderna. El país era todavía esencialmente agrícola, con herramientas y técnicas que databan del medioevo y ocupaban a casi tres cuartos de la población activa. La primera guerra mundial, transformó a España transitoriamente en 
exportadora de alimentos y hasta de manufacturas, pero su conclusión dio lugar a una situación que impidió nuevamente a España competir con las potencias industriales. Si a esto se le suma la crisis mundial de 1929, cuando las barras aduaneras cerraron el camino a la exportación agrícola, podemos hablar de una etapa crítica en la vida de esta nación. Este es el marco del desenvolvimiento del fútbol en España. A partir de ahora, para conocer este desarrollo del fútbol español, en relación a estos cambios mundiales en el este deporte, voy a nutrirme de una serie de trabajos sobre la temática, no solo en España, sino también en otras naciones, especialmente la Unión Soviética, con el fin de comparar y determinar la particularidad del caso.

\section{Los comienzos}

Durante el período abordado este deporte pasó de ser "una actividad de ocio propia de una reducida elite social, hasta llegar a convertirse a partir de la segunda mitad de los años veinte en un auténtico fenómeno de masas" (Sanjurjo, 2011: 2). Tal como se ha señalado en varios lugares el llamado deporte moderno se expande de la mano con el mercado mundial y el desarrollo planetario del sistema capitalista. "Los intercambios de capital, mercancías y trabajadores tuvieron como consecuencia los intercambios de ideas y prácticas deportivas" (Altuve, 2002: 29).

Todo comenzó cuando esa elite social, conservadora, en un momento del siglo XIX, va a intentar adoptar ciertas actividades de ocio "modernas". Estas, que sin duda entran en sintonía con los gustos de la burguesía comercial e industrial, ya se caracterizaban en buena medida por ingredientes de tipo lúdico y de actividad física y en muchos casos por su origen extranjero, mayormente inglés. Esta burguesía, "atrasada", al igual que en otros países vio en la actividad deportiva una forma de parecerse a los países más "avanzados" y será el sector que se va a apropiar inicialmente del fútbol. La demanda por actividades lúdicas modernas conlleva un deseo de actividades en espacios abiertos.

Así mismo, la penetración de nuevos espectáculos relacionados con la exhibición pública de habilidades físicas en las ciudades españolas puede verse también en el marco de desarrollo del ocio urbano moderno de finales del Ochocientos que irían cambiando los modelos de sociabilidad: el cinematógrafo, los espectáculos de variedades y el baile (Pujadas y Santacana, 2013: 150). 
En este cuadro entra el fútbol, que inicialmente era una actividad donde el eje estaba puesto en el placer y no en el resultado y era un producto de la industrialización y mutación de las ciudades, que acompañan la emergencia de esta burguesía urbana española (Pujadas y Santacana, 2013). Las transformaciones operadas durante este período van a alterar profundamente la fisonomía del fútbol, especialmente respecto a sus protagonistas. Los primeros deportistas y espectadores, que solo buscaban un entretenimiento por la diversión misma, actividad donde dominaban las nociones de fair play y sportmen, van a ser progresivamente reemplazados por sectores populares, que elevaron el fútbol a una de las actividades de ocio por excelencia, cuando este ya se había insertado en los principales tejido urbanos.

Tal como señalan Pujadas y Santacana (2013), especialmente a partir de los primeros años del siglo XX, el cambio se produjo producto de lo que los autores Ilaman "la mercantilización del deporte". Hacia el siglo XIX el deporte se desarrollaba en el marco de los clubes, y las cuotas sociales eran un factor imprescindible para el sostenimiento de la actividad. En este marco, todavía existían pocas diferencias entre la persona del socio y el jugador. El objetivo de las asociaciones civiles era el juego deportivo. Sin embargo, a medida que la popularidad de estas actividades y de sus clubes fue creciendo, comenzaron a aparecer otros actores, como los asociados no jugadores y los aficionados, lo cual fue generando rupturas y tensiones en el seno de los mismos clubes. El fin de esta indiferenciación va a marcar tensiones entre los jugadores y los dirigentes que en muchos casos derivo en escisiones y formación de nuevas asociaciones. Los límites iniciales del asociacionismo español van a ser progresivamente cruzados durante esta época. Siguiendo nuevamente a Pujadas y Santacana (2013), la comercialización de este deporte está relacionada con el desarrollo del artefacto urbano de ciertas ciudades como Barcelona y Madrid, que tiene lugar en paralelo con la necesidad de un espacio libre no urbano para la práctica de esta actividad. Tampoco se puede pasar por alto el contexto de florecimiento de otras actividades de ocio, de gran comercialización, entre las cuales el fútbol puede haber estado imitando esta tendencia.

Esta situación representó una gran crisis para los clubes que se vieron forzados a rotar permanentemente de sedes, empujados por el alza de precios (provocada por la urbanización) y la construcción urbana misma, que no dejaba espacio para la práctica e instaba a éxodos constantes en los equipos. Esto impuso, casi como medida de supervivencia, el pago de las entradas por parte del público, de forma relativamente temprana en 1902, como parte de las nuevas responsabilidades para mantener la subsistencia económica, en donde el dinero cobraba cada vez más importancia. 
La mercantilización incipiente del fútbol español, desde la perspectiva del pago de entrada a los recintos deportivos para el consumo de espectáculo, es por lo tanto un fenómeno vinculado a la primera década del siglo $\mathrm{xx}$ y a las necesidades económicas de una estructura social deportiva que se espectaculariza de forma rápida. La institucionalización del deporte, y por lo tanto las obligaciones económicas de los clubs con las federaciones, el mantenimiento social, los desplazamientos y el alquiler de instalaciones privadas más o menos fijas, son argumentos fácilmente reconocibles y utilizados por las sociedades como los causantes del inicio de una industria que se gesta en el escenario urbano (Pujadas y Santacana, 2013: 156).

En todo este proceso también juegan un rol los medios de comunicación que difunden, informan y construyen sentido en torno a esta práctica, contribuyendo a la masificación de esta actividad. De este modo, los principales elementos del desarrollo capitalista, es decir la conformación de ciudades, el proceso de industrialización, la intervención de las clases sociales propias del capitalismo (proletariado y burguesía), sumado a la posición española en el mercado internacional, van a dar fisonomía a estos primeros cambiantes años del deporte en este país. Pero el punto central aquí está en un elemento que va a marcar una época histórica en el fútbol mundial, que es el de la profesionalización de la actividad. Esta temática se desarrolló de forma retrasada respecto a Inglaterra, donde tuvo lugar rápidamente. Es que importación del fútbol en España se produjo en pleno proceso de comercialización y profesionalización de ese deporte, lo cual hacia lógico el desarrollo de un fenómeno similar en España, o por lo menos el despertar de un cierto debate al respecto.

\section{La profesionalización}

Nos situamos entre las décadas del 10 y del 20 del siglo XX. El fútbol se ha metido de lleno en el mercado de ocio. Compite con el cine, el teatro y las corridas de toros. Esto, siguiendo a Sanjurjo (2011) obligó a quienes conducían los destinos de este deporte a modificar drásticamente la gestion interna de los clubes, de sus federaciones y de las competencias existentes, con el fin de atraer el mayor número de espectadores y aumentar los beneficios económicos.

Hasta entonces los clubes existentes se nucleaban en torno a la Real Federación Española de Fútbol (RFEF), integrada por las diversas regionales, que sostuvo desde sus inicios un igualitarismo formal que definía su estructura interna y las relaciones de poder que la regían. 
Su función era coordinar las diferentes federaciones regionales, sin tener en cuenta el prestigio, fortaleza o importancia entre las mismas o la cantidad de clubes afiliados.

Este esquema durante los años veinte comenzará a mostrar claros signos de crisis al manifestarse importantes cambios en los intereses de buena parte de los elementos que la componen. Las federaciones regionales que contaban con los clubes más poderosos empezaron a reclamar un mayor protagonismo del que habían podido disfrutar hasta el momento. Esto provocó que progresivamente éstas traten de aumentar su representatividad dentro de las asambleas federativas para que su influencia se pueda ver reflejada en los organismos nacionales que controlaban el fútbol. La puja interna marcará la historia del fútbol español, porque incluirá una temática fundamental: el profesionalismo. La cuestión desató múltiples conflictos entre los clubes, que no hicieron otra cosa que pronunciar los cismas entre las distintas regionales caracterizadas por la denuncia del profesionalismo encubierto y acusaciones de favoritismo ante los entes reguladores.

Sanjurjo (2011) identifica hacia la década del veinte, tres tipos de clubes previos a la implantación del profesionalismo. Por un lado aquellos clubes, entre los que se encontraba el FC Barcelona, caracterizados por haber conseguido una estabilidad económica suficiente como para asegurar su futuro, logrando una fuerte identificación regional. El FC Barcelona, que llevaba unos cuantos años practicando un profesionalismo encubierto (1), será partidario de su regulación para de esta forma conseguir que los términos de los contratos pudieran quedar controlados por los propios clubes. Otros clubes eran mantenidos por uno o varios "padrinos" (2), y gracias a su aporte solventaban sus gastos y permitían la supervivencia de la institución, situación que podía ser inestable al depender del interés del financista. Por último estaba el grupo de clubes, entre los que destacaba el Athletic Bilbao, que se caracterizaban por el control de la cantera regional, lo cual le permitía conseguir los mejores jóvenes jugadores sin grandes gastos.

En la situación descripta en el apartado anterior, el jugador que había dejado de pertenecer a la elite social cuando el fútbol se transformó en un ocio de masas, abandonó los preceptos de origen aristocrático alejados del rendimiento y la competitividad y pasó a descubrir este deporte como un medio ideal con el que poder adquirir un mayor prestigio social y obtener dinero. Es decir, pasó a ser concebido como un potencial modo de vida y ascenso social.

La discusión de la profesionalización marcó los años veinte y se prolongó largamente en el tiempo. Si bien se aceptó hacia 1924, su reglamentación y puesta en práctica tuvo lugar recién en 1926. Los clubes discutían el control de la movilidad de la fuerza de trabajo, como un mecanismo de defensa para los clubes que entraban al mercado con menos poder que otros: los clubes tenían el derecho unilateral de retener a un futbolista cuyo contrato hubiese 
concluido, sin necesidad de un acuerdo entre ambas partes y a cambio únicamente de un modesto incremento salarial. Es decir restringía el movimiento de la mano de obra. Además se proponían establecer un techo salarial. La polémica acerca de la naturaleza de la situación fue tan amplia que llegó a incluir también la coexistencia con jugadores amateurs y las diferencias entre ambos (3). Tal como señala Sanjurjo,

\begin{abstract}
El proceso de "proletarización" del perfil social del futbolista sacará a la luz el verdadero conflicto de mentalidades que generaba entre los sectores más elitistas y tradicionales la progresiva pérdida de la antigua "exclusividad" que habían disfrutado hasta ese momento. La socialización del fútbol y su profesionalización chocaba con los valores fundamentales que habían dado forma al deporte amateur, lo que generará durante los años veinte una intensa polémica entre los defensores de la pureza del sport y los partidarios de la transformación de esta actividad en el primer deporte-espectáculo (2011: 9).
\end{abstract}

La llegada del profesionalismo, mejor dicho la larga discusión sobre el tema, legitimó parte de los cambios ya mencionados en la composición del deporte. El derecho a retención, en el marco de una medida de mercado como la profesionalización, implicaba no solo una suerte de intervencionismo superestructural (entre regulador), en el mercado de pases, sino una temática que afectaba profundamente a los deportistas, que parece también replicarse en otros países. En Argentina, el llamado "pase libre" fue uno de los motivos de los primeros reclamos de los futbolistas. En la Unión Soviética, donde regía un profesionalismo encubierto, el hecho de que un jugador cambiase regularmente de club, era visto como un síntoma de "deslealtad" y desarraigue respecto del colectivo (Edelman, 1993), y constituía uno de los principales temores de los líderes soviético acerca de instaurar abiertamente el profesionalismo.

En algún punto la discusión de la profesionalización es el colorario de un largo proceso que empezó un tiempo antes de su promulgación. En cierto momento algunos futbolistas comenzaron a considerarse imprescindibles y dejaron de pagar sus cuotas como socios de los clubes a los que representaban. Luego algunos comenzaron a recibir una serie de compensaciones materiales por jugar, que aumentaban en función del potencial de cada club que estaba relacionado con su capacidad de atraer fans y generar un espectáculo. Esta es una etapa, también común a muchas partes del mundo, denominada "amateurismo marrón" o profesionalismo encubierto. Se trata de una proto-profesionalización y tiene lugar durante la década de 1910 a un ritmo relativo a las posibilidades de cada institución.

Existe otro factor a la hora de entender el proceso de profesionalización: el del resultado deportivo. En algunos casos, una derrota deportiva impulsa a una reorganización de la 
estructura del fútbol, producto del análisis del "roce" con otros equipos o naciones. Es el caso soviético un ejemplo de ello, ya que una derrota de un combinado de los mejores equipos de Moscú frente al equipo francés profesional, Racing, va a desatar un debate y una reestructuración de la organización del fútbol en dicho Estado. En el caso español, el papel lo jugará un buen resultado, la medalla de plata en la Olimpíadas de 1920, que sacó al fútbol español de sus regionalismos y puso de relieve la dimensión de este juego, su dimensión y organización internacional, aunque la decepcionante actuación cuatro años después fue atribuida por algunos actores a la contaminación de los valores tradicionales del deporte, producto de la imparable profesionalización.

La profesionalización, reglamentada en 1926, determinó que un jugador profesional era aquel que recibía una remuneración o estipendio equivalente de cualquier clase, sin contar los recibidos para cubrir los gastos de viaje y hotel y aquellos otros generalmente autorizados como los de equipo, servicios de entrenador, masajista y asistencia en caso de lesión. De este modo se transformaba en un medio de vida. La profesionalización era solo para los deportistas, ya que la gestión de los clubes seguiría siendo amateur.

Una parte interesante de todo este proceso tiene que ver con la constitución del futbolista en tanto integrante de una clase social, obrera. Como fue señalado en párrafos anteriores, la movilidad de la "mano de obra" era limitada. Además la noción de "profesional" estuvo cargada de ambigüedades durante muchos años. Lo importante es que a estos deportistas no se los reconocía como trabajadores de los clubes (Sanjurjo, 2011). En los contratos de los jugadores se incluía una clausula obligatoria por la cual todas las partes se comprometían a resolver sus litigios en el marco federativo sin acudir a los tribunales de justicia ordinarios. Es decir que se eliminaba el carácter de clase en la relación entre las partes. Además el decreto que dio comienzo a la profesionalización, pronunciaba aún más la división ya obvia en el seno de los clubes entre jugadores y socios no practicantes, prohibiendo a los profesionales integrar la directiva de los clubes o federaciones. Esta división parecía pronunciar aún más el carácter de trabajadores enfrentados a un ente que los contrataba. Pero también los futbolistas eran separados de los otros trabajadores. Otra cláusula, bastante común también en el presente, prohibía a los deportistas formar parte de sociedades de clase o sindicatos opuestos a la disciplina de la RFEF, bajo la pena de perder su inscripción y no poder jugar en ningún club entre dos y cinco años.

Desde luego que está situación no desalentó a los intentos de los futbolistas de organizar un sindicato. Los catalanes sentaron precedente en 1924 (PREPROF), cuando los jugadores del FC Barcelona, crearon una sociedad mutual, para "defender los intereses de la clase ante la explotación de sus patronos" (Sanjurjo, 2011: 16) frente a la inminente reglamentación de la 
profesionalización. Esta no hizo más que radicalizar este proceso ya que los diarios hablan de que los deportistas de Madrid entraron en contacto con el sindicato socialista para crear la que inicialmente recibirá el nombre de Asociación Nacional de Trabajadores del Fútbol, y que más tarde se conocerá como la Unión Nacional de Jugadores del Fútbol. Es decir que al reconocimiento de su condición de trabajadores le agregan una concepción política acercándose a las filas partidarias obreras. Estas iniciativas que tuvieron avances y reflujos, marcan a fuego la experiencia de este colectivo de deportistas y son parte de su acervo común. La legislación que amenazaba eliminar la licencia profesional puede haber significado un desaliento a esta organización, pero la misma resurgiría con más fuerzas durante la guerra civil, especialmente en Barcelona, donde existió el Sindicato de Jugadores y Entrenadores de Fútbol.

Esta dinámica de reconocimiento como clase social, si bien la profesionalización de la actividad es una tendencia global no se encuentra en todos lados. Por ejemplo en el caso soviético, se emprendió una pseudo profesionalización (Edelman, 1993) "por arriba", es decir dictaminada por los dirigentes soviéticos deportivos, que si bien contó con el aval de los deportistas (no existían sindicatos), no los transformó ni formal ni informalmente en trabajadores, ya que su condición de asalariado, en el período que nos toca comparar estaba encubierta y debían tener un trabajo, que muchas veces era una "pantalla". De este modo los futbolistas se nutrían de diversos sectores sociales, al margen de todo lo que se pueda decir sobre la existencia de clases o no en la Unión Soviética. Situación distinta atravesaron los jugadores argentinos. Al igual que sus colegas españoles, también fueron atravesando un proceso de organización sindical, en forma de mutual, encararon huelgas que desencadenaron en la profesionalización (Frydenberg, 1999) y lucharon por convenios colectivos de trabajo.

Tanto el caso argentino como en el caso español, se da esta experiencia de lucha que va a integrarlos en una clase. Como señala Thompson:

\footnotetext{
Por clase entiendo un fenómeno histórico que unifica una serie de sucesos dispares y aparentemente desconectados, en lo que se refiere tanto a la materia prima de la experiencia, como a la conciencia. Y subrayo que se trata de un fenómeno histórico. No veo la clase como una "estructura", ni siquiera como una "categoría" sino como algo que tiene lugar de hecho (y se puede demostrar que ha ocurrido) en las relaciones humanas (1977: 1).
}

Las luchas, las idas y venidas, las categorías que forman a estos sujetos, son parte de este acervo, de esta experiencia común, que los configura como clase: "Al analizar procesos de 
enfrentamientos sociales podrá conocerse cuáles de las múltiples relaciones establecidas por los conjuntos de individuos, están en juego en un momento determinado y, por ende, si se están constituyendo en clase" (Iñigo Carrera, 2003: 8).

De la mano con la reglamentación de la profesionalización, y como algo inevitable se planteó la reorganización de la competencia futbolística. El aumento de los gastos, producto de la profesionalización, impulsará la necesidad de aumentar el número de partidos oficiales, dejando de lado una vieja competencia que implicaba campeonatos regionales, de poco interés para el público ya que enfrentaba equipos desequilibrados, dando lugar a posteriormente a una eliminatoria del Campeonato de España. La tendencia era hacia una liga más selectiva, con mejores y más populares jugadores, todo en aras de atraer a los espectadores. En tormo a esta reestructuración se sucedieron diversos debates y posicionamientos, entre los cuales se estructuraron los grupos conocidos en la prensa como "minimalistas" y "maximalistas". Las posiciones oscilaban entre una competencia solo entre aquellos que habían sido campeones de España y una competencia más amplia, siendo partidarios los primeros de una gestion autónoma de la RFEF, la cual se encargaría de las otras competencias y de la selección nacional.

Estas discusiones no dejaron de manifestarse a pesar del comienzo de la actividad oficial. El problema estaba en los equilibrios de poder. La ya mencionada estructuración igualitaria el voto equitativo por cada región, sería puesta en cuestión, por aquellas regiones que producto del desarrollo desigual de la actividad deportiva, como Cataluña, lograron mostrar un mayor peso específico y relevancia social, ya que albergaban a los clubes más importantes. Esta preeminencia no se veía reflejada dentro de la RFEF, que no contemplaba el "voto de calidad" al que aspiraban estas federaciones regionales. La llamada "mercantilización" del fútbol y la profesionalización no hizo más que consolidar estas ideas, a que fortificó la importancia de estos clubes y los transformó en el vehículo del éxito del conjunto del espectáculo. Recién en 1928 se llegó a un acuerdo:

Finalmente se consigue aprobar la participación directa de los clubes, diferenciando entre los que tendrían derecho propio como el FC Barcelona, Athletic de Bilbao, Real Madrid, Real Unión de Irún, Real Sociedad de San Sebastián y Arenas de Guecho, de aquellos que más tarde se acordará "que pertenezcan a la Nacional" como propietarios, haciendo referencia a un amplio número de clubes entre los que aparecerían entre otros el Celta de Vigo, Athletic de Madrid, Español de Barcelona, Sporting de Gijón, Sevilla, Valencia o el Racing de Santander. La asamblea incluirá finalmente a 36 equipos como miembros 
propietarios, que podrán participar en la toma de decisiones de la RFEF (Sanjurjo, 2011: 27).

\section{La guerra civil y el franquismo}

El nivel de exposición del deporte, especialmente del fútbol, durante la guerra civil fue sencillamente extraordinario. Se podría afirmar, que los clubes que se encontraban en tierras republicanas tuvieron un papel muy activo. La principal actividad desarrollada por los clubes estaba relacionada con eventos deportivos benéficos, que fueron de tal magnitud y en tal número que el propio el Comité Central de Milicias Antifascistas acabaría por intervenir para aconsejar la limitación de este tipo de festivales en favor de su racionalización y para evitar la competencia de público entre ellos (Pujadas, 2014). Estas actividades se caracterizaban por constantes manifestaciones antifascistas tanto del público como de sus protagonistas y una exaltación de los procesos sociales y políticos que tenían lugar en la retaguardia. Cuando los clubes, por cuestiones financieras se manifestaron a favor de retomar el calendario deportivo, en algunos casos como el FC Barcelona, una parte del costo de la entrada solventaría los gastos del frente. En la experiencia soviética, en el medio de la guerra revolucionaria contra los blancos, la esencia del deporte y la actividad física estaba vinculada con lo militar. Así se expresaba Lenin frente a la juventud comunista:

La educación física de la generación más joven es un elemento esencial en el sistema general de crianza de los jóvenes comunistas, destinada a la creación de personas armoniosamente desarrolladas, ciudadanos creativos de la sociedad comunista. Hoy, la educación física también tiene objetivos prácticos directos: 1) preparar a la gente joven para el trabajo; y 2) prepararlos para la defensa militar del poder soviético (Riordan, 1976: 159).

Esta experiencia como antecedente influyó sobre el caso español, ya que fue planteado por organizaciones de izquierda como el POUM y la CNT. Si bien las organizaciones socialistas y anarquistas habían tenido influencia sobre las asociaciones deportivas, con una fuerte carga ideológica en su actividad, recién cuando estalla el conflicto con las fuerzas franquistas se comienza a plantear esta cuestión. Dice Pujadas,

...la insurrección militar de julio y la guerra cambiaron la situación y dieron al deporte y a la educación física un protagonismo inédito, como pasó con tantas otras manifestaciones culturales y sociales. A nivel institucional, a creación de los primeros organismos deportivos 
públicos catalanes como el Instituto Catalán de Educación Física y Deportes (decreto de octubre 1936) reflejaron ese cambio y se vieron obligados a desarrollar la preparación física militar de las milicias y del Ejército Regular Republicano (1937), o bien a regularizar la actividad deportiva pública en tiempo de guerra (Comisariado de Educación Física), a pesar de no tener ninguna experiencia en ese terreno (2014: 306).

Pero sobre todo la guerra civil marcó la creación de una serie de organizaciones deportivas populares que luchaban por formas de sociabilidad alternativa. Abogaban por el acceso de las clases populares a la actividad deportiva, por su democratización, adoptando este movimiento el nombre de "deporte popular", además de las ya conocidas organizaciones deportivas, de alcance mundial, del socialismo (4) y el comunismo (5).

Sin embargo la contracara de este proceso de creatividad y efervescencia popular, la presentan los propios deportistas, que si bien combatieron y murieron en las trincheras (en muchos casos perjudicando la plantilla de sus equipos), en su campo laboral, optaron por obviar su condición de obreros. El control obrero que había emergido durante los primeros momentos de la guerra en muchas zonas controladas por los republicanos, tuvo su expresión en los clubes, en donde se crearon comités obreros. Sin embargo estos fueron integrados exclusivamente por el personal auxiliar de estas entidades, pero sin la presencia de los deportistas, con excepción de los boxeadores.

Por último, el deporte también fue parte de las disputas con el franquismo en el plano internacional, especialmente por la representación española en las competencias internacionales. "A los deportistas del mundo" escribían los republicanos en defensa de su causa y disputando todo tipo de competencias con otras naciones u organizaciones obreras solidarias, para intentar potenciar la difusión de la causa republicana.

Concluida la guerra civil y ya bajo el franquismo, el fútbol adoptó un carácter nacional, se volvió una cuestión de Estado, sometiéndose a la intervención del mismo y sus organizaciones políticas (la Falange Española Tradicionalista), las cuales exigieron a las entidades adhesión explicita al régimen. El deporte se transformó en una vía para la integración nacional, la socialización política de los jóvenes y lógicamente un vehículo para la propaganda ideológica, ya que la idea de la unidad nacional, pasa por alto las contradicciones tanto a niveles sociales como regional, existentes en la nación española.

La utilización del fútbol como un engranaje más en el funcionamiento de este Nuevo Estado, su reestructuración en torno a ideas de corte fascista como la obediencia, el sometimiento y la disciplina militar, también saldaba cuentas con el pasado. De acuerdo con Llopis Goig, un reconocido periodistas afirmaba que: 
El fútbol era durante la Segunda República una orgía roja de las más pequeñas pasiones regionales de las más viles (...) casi todo el mundo era separatista -y grosero- frente a un match para el Campeonato de España. El bizcaitarrismo se daba tanto en los graderíos de San Mamés como en la tribuna de Chamartín. En la mayoría de los casos, el madridista era un bizcaitarra de Madrid; es decir, un localista retrasado mental frente a los límites nacionales (s.f.: 4).

En este cuadro los clubes, al transformarse en un mero apéndice de la política estatal perdieron su naturaleza privada y su capacidad de autogobierno, perdiendo los socios su papel protagónico, convirtiéndose en meros abonados a un espectáculo. Sin embargo, el fútbol retomo rápidamente sus niveles de asistencia y socios previos a la guerra y experimentó un crecimiento muy importante. En el plano internacional fueron creados diversos órganos como el Consejo Nacional de Deportes y finalmente fue reconocida por la FIFA, la Federación Nacional de España como la legitima representante de dicha nación.

\begin{abstract}
Como en el resto de las instancias oficiales, la Federación nombró comités depuradores para sancionar a jugadores, entrena-dores y árbitros que hubiesen mostrado su lealtad a la República. Troncoso advirtió además que las federaciones y los clubes de fútbol habían dejado de existir como entes autónomos, y exigió a los futbolistas virtudes análogas a las de un soldado (Calleja, 2014: 283).
\end{abstract}

\title{
Palabras finales
}

Partir de la afirmación de Archetti para comenzar este estudio permite dimensionar el fenómeno que atraviesa el deporte español por aquellos años. Los dramas de esta sociedad se manifiestan de forma muy clara en el fútbol y se entrelazan con las características políticas, sociales y económicas de la nación durante ese periodo. Por un lado el regionalismo, que aparece como un motor del proceso de profesionalización y reorganización de este deporte, expresa la lucha por la autonomía de las distintas nacionalidades contenidas en el Estado español. Los sucesivos reagrupamientos, frentes comunes en torno a cómo organizar la profesionalización o la nueva liga manifiestan el "equilibrio de tensiones entre opuestos en todo un complejo de polaridades interdependientes" (Dunning, 249) entre las que se encuentran las distintas autoridades, espectadores, jugadores, equipos, etcétera. Pero como explica el mismo 
autor, las actividades deportivas están relacionadas con una estructura de interdependencias más amplias que incluye al conjunto de la sociedad, su tejido social.

La disputas en torno a la profesionalización, que ponen de manifiesto la pérdida del privilegio deportivo de las clases altas, frente a las necesidades de modernización producto de la masificación del juego, ilustran un proceso en que lo "viejo" (el deporte, por el deporte mismo) comienza a morir y se resiste a que llegue su hora, así como las luchas en torno a los derechos de los trabajadores y sus constitución en tanto tales. Tal como señala Dunning (2222) este proceso fue una acción colectiva de un sector que incursionaba en un campo otrora exclusivo de las capas más altas de la sociedad. De este modo, las tensiones en torno a la profesionalización, siguiendo esta propuesta, decantan en los argumentos de estos sectores sociales en defensa de los valores del amateurismo, en una acción defensiva en torno a un espacio social que consideraban exclusivo. Otra reflexión interesante del autor a la hora de hablar de la emergencia del deporte moderno, se ponía de manifiesto la tendencia a la competencia, al fin del deporte por el deporte mismo, la caída de los valores que lo caracterizaban. Al mismo tiempo la aparición de masas de espectadores y un deporte orientado hacia su satisfacción. Un proceso de "desaficionismo" del juego.

Así sintetiza Sanjurjo la situación española:

\begin{abstract}
Aquellos que veían el fútbol como una pasión y que no necesitaban este deporte como medio de vida, se opondrán radicalmente a la nueva deriva del profesionalismo, mostrando su disgusto por la pérdida de un protagonismo que habían acaparado durante tanto tiempo. Su crítica se centraba en la dirección plebeya que el fútbol estaba adquiriendo y en su progresiva utilización como instrumento de ascenso social bajo parámetros mercantiles. Frente a los anteriores, surgirá un sector social más pragmático que entenderá y aceptará la profesionalización del fútbol, conscientes que era un fenómeno imparable y que exigiría una readaptación de todos sus elementos ante el nuevo contexto del deporte-espectáculo (2011: 28).
\end{abstract}

Estos dramas propios de una sociedad de las características que Broue le atribuye a esta nación, van a ser negadas, rechazadas por el discurso y utilización franquistas del deporte. Tanto la lucha de los futbolistas en calidad de trabajadores como las pujas entre las regiones que se manifestaron en el porvenir del fútbol durante el periodo estudiado, van a ser rechazadas por el nuevo deporte bajo el franquismo. Estas que aparecen como unas de las tantas polaridades del deporte moderno, van a ser condenadas por el nuevo régimen, sometiendo esta actividad deportiva a sus designios. 


\section{Notas}

(1) Un régimen de pagos encubiertos de los clubes hacia los jugadores.

(2) Se trata de sujetos de gran importancia social que solventaban la actividad de una institución. Podía provenir del ámbito empresarial, aunque también político, espacios que eran utilizados para obtener facilidades a favor de sus clubes.

(3) "Pero la principal dificultad radicaba en cómo demostrar fehacientemente esta situación irregular, llegando a provocar que algunos periodistas se planteen la necesidad de separar a los jugadores profesionales de los amateurs, porque "siendo el profesionalismo la especialización, significa, por tanto, un adelanto en la práctica del deporte, al que nos lleva automáticamente el desarrollo del mismo, y es lógico separar a los que luchan en desigualdad de condiciones" (Sanjurjo, 2011: 11).

(4) Internacional Deportiva Obrera.

(5) Internacional Roja del Deporte y la Gimnasia.

\section{Bibliografía}

Altuve, E. (2002). Deporte. Modelo perfecto de Globalización. Maracaibo, Venezuela: CEELA. Archetti, E. (1985). Fútbol y Ethos. Buenos Aires, Argentina: FLACSO.

Broue, P. y Témime, E. (1962). La Revolución y la Guerra de España. México D.F, México: Biblioteca Actual.

Calleja, E. (2014). El Real Madrid, ¿"equipo de España"? Fútbol e identidades durante el franquismo. Política y Sociedad, 51(2), pp. 275-296.

Dunning, E. (2014). La dinámica del deporte moderno: notas sobre la búsqueda de triunfos y la importancia social del deporte. En Elias, N.y Dunning, E. Deporte y ocio en el proceso de la civilización (pp. 247-269). México DF, México: FCE.

Edelman, R. (1993). A History of Spectator Sports in the USSR. Nueva York, EEUU: Oxford University Press.

Elias, N. (2014). La génesis del deporte como problema sociológico. En Elias, N. y Dunning, E. Deporte y ocio en el proceso de la civilización (pp. 157-184). México DF, México: FCE.

Frydenberg, J. (1999). El nacimiento del fútbol profesional argentino: resultado inesperado de una huelga de jugadores. Efdeportes. Recuperado de http://www.efdeportes.com/efd17/futpro.htm

Frydenberg, J. (2011). Historia social del fútbol: del amateurismo a la profesionalización. Buenos Aires, Argentina: Siglo veintiuno editores. 
Iñigo Carrera, N. (2003). El concepto de clase obrera. Recuperado de http://www.iisg.nl/labouragain/documents/inigocarrera.pdf.

Llopis Goig, R. (s.f). Claves etnoterritoriales de la historia del fútbol español, Cafyd. Recuperado de www.cafyd.com/HistDeporte/htm/pdf/2-24.pdf

Pujadas, X. (2014). Entre estadios y trincheras. El deporte y la Guerra Civil en Cataluña (19361939). En Pujadas, X. (coord.). Atletas y ciudadanos. Historia social del deporte en España 1870-2010 (pp. 302-306). Madrid, España: Alianza Editorial.

Pujadas, X. y Santacana, C. (2013). La mercantilización del ocio deportivo en España. El caso del fútbol 1900-1928. Fundación Instituto de Historia Social, 41, pp 147-167.

Riordan, J. (1976). Marx, Lenin and Physical Culture. Journal of Sport History, 3, pp 152-161.

Sanjurjo, J. (2011). La mercantilización del fútbol español en los años veinte: de la implantación del profesionalismo al nacimiento del campeonato nacional de liga. Esporte e Sociedade, 6, pp 1-30.

Thompson, E. (1989). La formación de la clase obrera en Inglaterra. Barcelona, España: Crítica. 